\title{
Isometric was better than Isotonic Neck Stretching to Reduce Pain and Creatinine Kinease in Non-specific Neck Pain in Caring Students of Swadhyaseni Middle High School, Badung, Bali
}

\author{
Thomas Eko Purwata*, I. Komang Sri Mahendra Putra, I. G. N. Purna Putra and I. Putu Eka Widyadharma \\ Faculty of Medicine, Department of Neurology, Udayana University/Sanglah Hospital, Bali, Indonesia; \\ thomaseko2000@yahoo.com
}

\begin{abstract}
Neck pain is frequently complained by workers with static neck position. Complaints accompanied by discomfort around the neck muscles without any sensory disturbances or radiating pain, without specific causes such as history of neck trauma, neck malignancy or anatomical structural abnormalities. The samples were taken from carving students of Swadhyaya Middle School Badung. We evaluated 18 students divided into 2 groups: one with isometric neck stretches and another with isotonic neck stretches, 9 subjects each. The paired t-test found the number of decrease in NPRS in the isometric group was greater than isotonic group and the difference was statistically significant. The results of the unpaired student $t$-test obtained a mean decrease in NPRS in the isometric group greater than isotonic with a value of $P=0.00(p<0.01)$. Creatine kinase levels in the isometric group showed no significant difference, whereas creatine kinase levels in the isotonic group showed that the increase was not significantly different, the results of the independent student $t$ test showed values not significantly different from the isometric and isotonic groups with the intention of $p=0.284(p>0.01)$. These results indicate isometric stretching is more effective in reducing the scale of NPRS pain in non-specific neck pain compared to isotonic stretches, isometric and isotonic stretching techniques are equally less effective in reducing creatine kinase levels.
\end{abstract}

Keywords: Creatine Kinase, Isometric, Isotonic, Nonspecific Neck Pain, Pain Scale

\section{Background}

Neck pain is a complaint that is often felt by workers, especially workers with a certain neck position in a long enough period of time resulting in excessive contraction of the neck muscles. Various types of work can lead to complaints of neck pain especially for workers with wrong body position that affects the position of the neck resulting in non-specific neck pain.

Approximately 54\% of individuals in the world have complained of neck pain in a period of six months and this incidence continues to increase in prevalence around $6 \%$ to $22 \%$ and increases in the old age group of around $38 \%$. The costs incurred by workers for treatment of neck pain are sufficiently high. Research showed that the prevalence of musculoskeletal pain in the neck in the community for one year was $40 \%$ and more prevalent in women, while the prevalence of neck pain in workers ranges from 6-76 \% and mostly in women $\frac{1-3}{}$.
The research conducted by Nemade ${ }^{4}$ obtained musculoskeletal complaints in the form of neck pain in brick workers in India by 14\% from 159 workers, while the research conducted by Sihawong et al. .5 in 567 office workers provided stretching training in the treatment group totaling 285 people and without treatment of neck stretching in 282 people as the control group, resulted in a decrease in the incidence of neck pain in office workers who received neck stretch treatment $t^{4,5}$.

Body posture and muscle contraction during work can cause trauma to various aspects of the musculoskeletal system, especially the vertebral column muscles. Forward head posture occurs due to increased dorsal spinal kyphosis, where the head is in front of the center of gravity so that the neck load in Broocrease. Dropping shoulders affect the cervical spine, and excessive hyperextension or hyperflexion in the neck also affect the incidence of neck pain in workers ${ }^{6}$.

Research conducted by Nejati et al. ${ }^{7}$ in Iran on 46 people without neck pain and 55 people with neck pain who worked in offices with computers found that head forward posture 
correlated with the incidence of neck pain while shoulder posture had no effect ${ }^{7}$. Research conducted by Mufti on the craftsmen of songket weaving in Pandai Singkek ${ }^{8}$, West Sumatra regarding the study of workers' posture showed that all workers complained of neck pain (100\%), followed by upper back pain $\stackrel{8}{-}$

Research conducted by Palmer et al. ${ }^{9}$ in England, Scotland, and Wales in 12,907 respondents aged 16-64 years showed that people who worked with upper arms and shoulders more than one hour per day had a significant association with the onset of neck pain, while a prospective study conducted by Ariens et al. found that workers who worked in a sitting position with a static neck position when working was a risk factor for neck pain?.

Excessive muscle activity will cause damage to muscle cells. This condition is evidenced by high levels of Creatine Kinase (CK) in blood serum. After heavy activity the muscles need either active or passive recovery. Active recovery by mild stretches can reduce adverse metabolic results of muscles including reducing levels of creatine kinase and lactic acid in the muscles ${ }^{10}$.

Stretching is one way that is often done to reduce discomfort due to pain in the muscles of the neck and shoulders. Stretching provides benefits to strengthen the muscles of the neck and shoulders, increases flexibility and movement of the neck muscles, increases blood flow to the muscles, increases production energy, reduce pain and are relatively easy to do and do not require special time and place. Research conducted by Sowmya et al. in patients with non-specific chronic neck pain showed an isometric stretching technique as effective as dynamic or isotonic stretching to reduce non-specific neck pain ${ }^{11}$. Gross et al. obtained moderate results in patients with non-specific neck pain who were given isometric stretches ${ }^{12}$.

Neck pain can be caused by all components of the neck starting from the musculoskeletal system and nervous system. Neck pain in workers is generally more often caused by musculoskeletal disorders both due to tension and excessive contraction of muscles and ligaments around the neck and giving symptoms of pain and neck stiffness accompanied by muscular pain around the neck.

Neck pain experienced by workers often involves disorders of the musculoskeletal system, this neck pain according to the pathophysiological process including mechanical neck pain or axial neck pain or often called non-specific neck pain as there is no underlying disease or structural anatomic disorder. The symptoms that often accompany non-specific neck pain are stiffness in one side or both sides of the neck, and pain radiating to the head. Nonspecific neck pain is caused purely by the structure of the muscles or musculoskeletal system in the neck and is often related to body posture or static neck position at work and excessive neck muscle workload when working for a certain period of time $e^{2,13}$.

\section{Objectives}

To find out the more effective stretching technique between isometric and isotonic to reduce pain scale and creatine kinase levels in non-specific neck pain.

\section{Materials and Methods}

This research is an experimental study with a pretest-posttest control group design. Subjects was taken from students of Swadhyaya Middle Carving School Badung, Bali from November 2016 to December 2016 by simple random sampling method. Data collection was done by filling in the questionnaire by the subjects and by neurological clinical examinations.

Inclusion criteria were: students following carving art training activities; non-specific neck pain with NPRS 1-6 (mild - moderate); not taking or undergoing any pain relief therapy; filling in inform consent. The exclusion criteria were: neck trauma; history of neck surgery; severe neck pain; regular relaxation; radiculopathy; history of heart disease and hypertension.

The total sample size needed in this study was 18 people with details of 9 as isometric groups and 9 as isotonic groups.

The analysis and presentation of the data used were as follows:

- Descriptive analysis to give an idea of the characteristics of the data obtained in this study such as age, sex, weight, height, pain scale and creatin kinase levels pre- and post- treatment.

- The normality test on numerical data (NPRS) using the Shapiro Wilk test because the number of samples is less than 50 .

- Bivariate analysis to compare the mean reduction in NPRS and creatine kinase before and after stretching. Numerical scale data in both groups tested using the Student paired $\mathrm{t}$-test and unpaired $\mathrm{t}$-test when the data was distributed normally and the Wilcoxon test if the data were not distributed normally. The level of significance was set as $\mathrm{p}$ value $<0.01$.

- If drop out $>20 \%$ then analysis of intention to threat is carried out. All data were analyzed using the SPSS 20.0 for Windows. 


\section{Results}

\subsection{Basic Characteristics of Research Subjects}

Based on the sex category, all subjects, 9 in each group, were male (100\%). The mean of the overall age category was 14.76 years. The average age of the isometric group is 14.44 years and the average age of the isotonic group is 15.11 years, the mean weight of the isometric group is $47.44 \pm 1.74$ and isotonic is $47.22 \pm 1.64$ while the mean height of the isometric group is $150,00 \pm 2.42$ and the isotonic group is $149.00 \pm 3,201$. The initial mean NPRS in isometric group was $4.89 \pm 0.601$ and after 4 weeks following the isometric stretching exercise the scale of pain dropped to $1.89 \pm 0.601$. The initial NPRS in the isotonic group was $4.67 \pm 0.707$ and the pain scale at the end of the exercise was $3.44 \pm 0.527$. The initial CK level in the isometric group was $170.11 \pm 41.963$ and at the end of the exercise was $156.00 \pm 31,389$ while the initial level of $\mathrm{CK}$ in the isotonic group was $170.67 \pm 52.557$ and at the end of the exercise was $177.78 \pm 62.532$. The basic characteristics of the research subjects are presented in (Table 1 ).

\subsection{Effects of Isometric and Isotonic Stretching on Decreasing the Scale of NPRS Pain}

The effect of stretching on decreasing the NPRS pain scale in patients with non-specific neck pain was assessed by comparing the mean reduction in NPRS between isometric groups and isotonic groups after stretching exercises for 4 weeks. Normality tests on the NPRS reduction in both groups using the Shapiro Wilk test showed normal distribution. The analysis is presented in the following (Tables 2 and 3).

Table 1. Basic characteristics of subjects

\begin{tabular}{|l|l|l|}
\hline Variable & isometric $(\mathbf{n}=\mathbf{9})$ & isotonic $(\mathbf{n}=9)$ \\
\hline Mean age (years) & $14.44 \pm 0,527$ & $14,78 \pm 0,441$ \\
\hline Sex (male) & $9(100 \%)$ & $9(100 \%)$ \\
\hline Weight (kg) & $47,44 \pm 1,74$ & $47,22 \pm 1,64$ \\
Height (cm) & $150,11 \pm 2,42$ & $149,00 \pm 3,20$ \\
Initial (pre-treatment) NPRS & $4,89 \pm 0,601$ & $4,67 \pm 0,707$ \\
Final (post-treatment) NPRS & $1,89 \pm 0,601$ & $3,44 \pm 0,527$ \\
Initial (pre-treatment) CK (U/L) & $170,11 \pm 41,963$ & $170,67 \pm 52,557$ \\
Initial (pre-treatment) CK (U/L) & $156,00 \pm 31,389$ & $177,78 \pm 62,532$ \\
\hline
\end{tabular}

Table 2. Bivariate analysis of Student's paired t-test in mean decrease of NPRS

\begin{tabular}{|l|l|l|l|l|}
\hline Group & Initial mean NPRS & Final mean NPRS & Percentage & p-value \\
\hline Isometric & $4,89 \pm 0,601$ & $1,89 \pm 0,601$ & $61,34 \%$ & 0,000 \\
\hline Isotonic & $4,67 \pm 0,707$ & $3,44 \pm 0,527$ & $26,39 \%$ & 0,000 \\
\hline
\end{tabular}

Table 3. Bivariate analysis of NPRS using unpaired t-test

\begin{tabular}{|l|l|l|}
\hline Group & Mean NPRS decrease & p-value \\
\hline Isometric & $3,00 \pm 0,707$ & 0,000 \\
\hline Isotonic & $1,22 \pm 0,441$ & \\
\hline
\end{tabular}

\subsection{Effects of Isometric and Isotonic Stretches on Decreasing Creatine Kinase Levels}

The decrease in creatine kinase levels in the study subjects was calculated by comparing the mean creatine kinase levels before and after stretching exercise. The normality test using the Shapiro Wilk test showed that the data were distributed normally. The analysis results are presented in the following (Tables 4 and 5).

Table 4. Bivariate analysis of Student's paired t-test of mean decrease of CK

\begin{tabular}{|l|l|l|l|l|}
\hline Group & Initial mean CK & Final mean CK & Percentage & p-value \\
\hline Isometric & $170,11 \pm 41,963$ & $156,00 \pm 31,389$ & $8,29 \%$ & 0,030 \\
\hline Isotonic & $170,67 \pm 52,557$ & $177,78 \pm 62,532$ & $4.16 \%$ & 0,709 \\
\hline
\end{tabular}


Table 5. Bivariate analysis of unpaired t-test of decrease in CK level

\begin{tabular}{|l|l|l|}
\hline Group & Mean decrease in CK level & p-value \\
\hline Isometric & $14,11 \pm 16,058$ & 0,284 \\
\hline Isotonic & $7,11 \pm 55,151$ & \\
\hline
\end{tabular}

\section{Discussion}

\subsection{Basic Characteristics of Research Subjects}

The subjects of this study were obtained from the students of Swadhyaya Middle School, Badung, Bali who experienced nonspecific neck pain, selected by using the LEEDS questionnaire Assessment of Neuropathic Symptoms and Signs (LANNS) and simple randomization with coin toss on the students meeting inclusion criteria. A total of 18 students were divided into 9 students as treatment groups with isometric stretches and 9 students as a control group with isotonic stretches.

All subjects were male due to the school curriculum dictated that all male student in the Swadhyaya Middle School taking carving class while the female students taking the sewing class. The average age of the whole group in this study was 14.78 years, with the average age of isometric groups being $14.44 \pm$ 0.527 and the age of the Isotonic group was $14.78 \pm 0.441$ with the lowest age of 14 years and the highest age of 15 years. The mean age of the isometric and isotonic groups did not differ statistically with a value of $\mathrm{p}=0.165(\mathrm{p}>0.01)$.

The mean weight of the isometric and isotonic groups was not significantly different with the value of $\mathrm{p}=0.784(\mathrm{p}>0.01)$ and the mean height of the isometric and isotonic groups also did not differ statistically with a value of $p=0.419(p>0.01)$. Data on the characteristics of the research subjects such as age, weight and height did not differ between isometric groups and isotonic groups because all subjects in this study were attending grade 3 and had never stayed in classes, participating in arts activities. carve regularly at school 3 times a week.

\subsection{Effects of Isometric and Isotonic Stretching on Decreasing the scale of NPRS Pain}

The mean NPRS in the isometric group at the start of stretching was $4.89 \pm 0.601$ and at the end of the exercise was $1.89 \pm 0.601$. This result indicated a decrease in the mean value of $61.34 \%$ and this was statistically significant $(p=0,000)$. In the isotonic group, the initial mean of NPRS was $4.67 \pm 0.707$ and the final mean was $3.67 \pm 0.707$. The decrease in the mean NPRS value in the isotonic group was $26.39 \%$ and statistically significant $(\mathrm{p}=0.000)$. To determine the effectiveness between isometric and isotonic stretching, an independent student t-test was performed with the results of the mean reduction in the isometric group $3.00 \pm 0.707$ and the mean decrease in the isotonic group $1.22 \pm 0.441(\mathrm{p}=0.000)$. This indicated that the isometric stretching technique was more effective in reducing the scale of NPRS pain in patients with non-specific neck pain compared to the isotonic stretching technique. The result of this study was consistent with the results of a study conducted by Gupta et al. ${ }^{14}$ who found a decrease in the pain scale of both isometric and isotonic groups in patients with acute nonspecific neck pain; in the study the pain scale decreased in an average of 4 or around 72 .

The isometric group showed better result compared to the isotonic group. This might be due to the mechanism of the isometric stretch to return muscle fibers, actin and myosin, to the normal position after overlapping between actin and myosin fibers due to excessive muscle contraction (cross link). The condition of cross links in muscle fibers will irritate the A delta and $\mathrm{C}$ fibers that is responsible for pain. The return of actin and myosin fibers to their normal position helps repair irregular muscle fibers and will cause the muscles to relax. There is an endogenous release of analgesic so that pain can be reduced or eliminated. In the isotonic group, the degree of improvement was not as high, possibly due to the muscle movements involving varying muscle lengths along with movement of the joints around the neck so metabolism is greater, and more energy is needed. This stretch can help return the position of muscle fiber fibers in a normal position, however the length of muscles changes so that normal conditions are achieved in only a short duration because the muscles contract again.

\subsection{Effects of Isometric and Isotonic Stretches on Decreasing Creatine Kinase Levels}

The initial mean creatine kinase level in the isometric group was $170.11 \pm 41.963$ and after 4 weeks stretching was $156.00 \pm 31,389$ with a decrease in creatine kinase level of $8.29 \%$. This result was not statistically significant with a $p$ value $=0.341$. In the isotonic group, the initial mean creatine kinase level was $170.67 \pm 52.557$ and after stretching was $177.78 \pm 62.532$; the results interestingly showed an increase in the average CK level of $5.46 \%$, even though it was not statistically significant $(\mathrm{p}=0.629)$. To determine the effectiveness of isometric and isotonic stretching to reduce CK levels, an independent Student $\mathrm{t}$-test was done and the CK reduction in isotonic groups was found to be an average $11 \pm 16,058$ and the mean increase in CK in the isotonic group was $7.11 \pm 55.151$ 
with a value of $\mathrm{p}=0.285$ ( $\mathrm{p}>0.01)$. It can be concluded that both isometric and isotonic stretches were equally ineffective in reducing CK levels in non-specific neck pain. This might be explained by the fact that CK level in the blood was influenced by genetics, sex, age and activity. Because the activity of the subject of research outside the school varied with different intensities so it was possible that the serum CK level differed essentially. Same reasoning may be applied to the increase in the average $\mathrm{CK}$ level in the isotonic group, in addition to the mechanism by which isotonic stretching requires greater energy and muscle contraction so that muscle metabolism and energy consumption increases, thus the elevated CK level. All subjects in this study had CK levels below $270 \mathrm{U} / \mathrm{L}$ (ages 13-17 years) which showed no muscle damage.

Research conducted by Clarkson et al. $\frac{15}{}$ obtained an average increase in CK of 34\% in the isometric group and 37.6\% in the isotonic group. The research conducted by Agha-Alinejad et al. ${ }^{16}$ on 28 students who took a physical exercise program to determine the effect of isotonic and isometric stretches after certain activities had increased CK levels. Baird et al. ${ }^{10}$ obtained different results, namely a mild isometric stretching exercise program reduced muscle damage and CK levels compared to heavy physical exercise.

The weakness in this study is the small number of samples and the short duration of the study. Another disadvantage is that the subjects in this study were not quarantined so it was difficult to monitor activities carried out outside of school whereas CK levels in the blood were strongly influenced by the activity of the body muscles. Another disadvantage is that in this study the force or energy released during stretching could not be measured with certainty so that the force or energy released by each subject varied.

The strength of this study lies in the homogeneous sample of school children so that samples were easily followed up and bias could be avoided. Stretching techniques were trained by experienced physiotherapy instructors and stretching exercises were carried out in separate spaces between isometric and isotonic groups so as to minimize errors and mimic each other's movements in stretching. All subjects were not exercised or underwent exercise in school during the participation period, thus minimized the influence of other physical activities outside of the neck stretching activities.

\section{Conclusion}

Based on the results of this study, it can be concluded that isometric neck stretching is better than isotonic neck stretching to reduce pain scale in patients with non-specific neck pain and both isometric and isotonic stretches are equally ineffective in reducing creatine kinase in non-specific neck pain. Stretching isometric techniques can be used as one of the non-pharmacological management modalities to reduce nonspecific neck pain. However, further research should include a larger number of samples, involving female subjects, longer training duration and more specific biomarker measurements for inflammation such as interleukin and other muscle damage biomarkers such as myoglobin, lactate dehydrogenase (LDH) and aspartate aminotransferase (AST).

\section{References}

1. Ariens GA, van Mechelen W, Bongers PM, Bouter LM, van der Wal G. Psychosocial risk factors for neck pain: A systematic review. American Journal of Industrial Medicine. 2001; 39:180-93. https://doi.org/10.1002/1097-0274(200102)39:2<180:: AID-AJIM1005>3.0.CO;2-\#

2. Giannoula T, Roy R, Win D, Paul VN. Nonspecific neck pain and evidence based practice. European scientific Journals. 2013; 9:6.

3. Masum SB, Haqe M, Haque M, Islam MS. Prevalence of neck pain and associated factors among office workers. The American Journals of Innovative Research and Applied Sciences. 2014; 1:4.

4. Nemade VV. Musculoskeletal disorders in brick client workers. Incidence and Effectiveness of Workers Education Programe. 2014; 3:3.

5. Sihawong R, Janwantanakul P, Sitthipornvorakul E, Pensri P. Exercise therapy for office workers with nonspecific neck pain: a systematic review. Journal of Manipulative and Physiological Therapeutics. 2011; 34:62-71. https://doi.org/10.1016/j. jmpt.2010.11.005. PMid:21237409

6. Brook P, Bogduk N. Evidence based management of acute musculoskletal pain. The National Health and Medical Research Council, First Edition; 2003. p. 14.

7. Nejati P, Lotfian S, Moezy A, Nejati M. The study of correlation between forward head posture and neck pain in Iranian office workers. International Journal of Occupational Medicine and Environmental Health. 2015; 28:295-303. https://doi.org/10.13075/ijomeh.1896.00352

8. Mufti D, Suryani E, Sari N. Evaluation of work posture among Songket fabric craftsmen at Pandai Sikek. Scientific Industrial Technique Journal. 2013; 12:63-71.

9. Palmer KT, Walker-Bone K, Griffin MJ, Syddall H, Pannett B, Coggon D, Cooper C. Prevalence and occupational associations of neck pain in the British population. Scandinavian Journal of Work, Environment \& Health. 2001; 27:49-56. https://doi.org/10.5271/sjweh.586. PMid:11266146

10. Baird MF, Graham SM, Baker JS, Bickerstaff GF.0 Creatinekinase- and exercise-related muscle damage implications for muscle performance and recovery. Journal of Nutrition and Metabolism. 2012;2012:13. https://doi.org/10.1155/2012/960363. PMid:22288008. PMCid:PMC3263635. 
11. M.V S. Isometric neck exercises versus dynamic neck exercises in chronic neck pain; 2014.

12. Gross A, Kay TM, Paquin JP, Blanchette S, Lalonde P, Christe $\mathrm{T}$, et al. Exercises for mechanical neck disorders. Cochrane Database of Systematic Reviews. 2015. https://doi.org/10.1002/14651858.CD004250.pub5

13. Binder A. The diagnosis and treatment of nonspecific neck pain and whiplash. Europa Medicophysica. 2007; 43:79-89. PMid:17369782

14. Gupta S, Jaiswal P, Chhabra D. A comparative study between postisometric relaxation and isometric exercises in non-specific neck pain. 2012.
15. Clarkson PM, Byrnes WC, McCormick KM, Turcotte LP, White JS. Muscle soreness and serum creatine kinase activity following isometric, eccentric, and concentric exercise. International Journal of Sports Medicine. 1986; 7:152-5. https://doi.org/10.1055/s-2008-1025753. PMid:3733311

16. Agha-Alinejad H, Shamsi M, Azarbayjan M, Rahimi M, Jafarabadi MA, Tofighi L, Mirani M. The effects of active recovery on serum IL-6, IL-8, IL-10 and CK concentrations after eccentric strenuous exercise in active female. Iranian Journal of Endocrinology and Metabolism. 2010; 11(5):553-60. 